\title{
Existence and Global Stability of a Periodic Solution for Discrete-Time Cellular Neural Networks
}

\author{
Haijian Shao, Haikun Wei, and Haoxiang Wang \\ Department of Automation, Southeast University, Nanjing, Jiangsu 210096, China \\ Correspondence should be addressed to Haijian Shao, shaohaijian2012@gmail.com
}

Received 9 July 2012; Revised 16 September 2012; Accepted 15 October 2012

Academic Editor: Kwok-Wo Wong

Copyright (c) 2012 Haijian Shao et al. This is an open access article distributed under the Creative Commons Attribution License, which permits unrestricted use, distribution, and reproduction in any medium, provided the original work is properly cited.

A novel sufficient condition is developed to obtain the discrete-time analogues of cellular neural network (CNN) with periodic coefficients in the three-dimensional space. Existence and global stability of a periodic solution for the discrete-time cellular neural network (DT-CNN) are analysed by utilizing continuation theorem of coincidence degree theory and Lyapunov stability theory, respectively. In addition, an illustrative numerical example is presented to verify the effectiveness of the proposed results.

\section{Introduction}

Cellular neural networks (CNNs) are the basis of both discrete-time cellular neural networks (DT-CNNs) [1] and the cellular neural networks universal machine (CNNs-UM). The dynamical behaviour of Chua and Yang cellular neural network (CY-CNN) is given by the state equation

$$
\begin{gathered}
C \frac{\mathrm{d} x_{i j}}{\mathrm{~d} t}=-\frac{1}{R} x_{i j}+\sum_{C(k, l) \in N r(i j)} A_{k l} y_{k l}+\sum_{C(k, l) \in N r(i j)} B_{k l} u_{k l}+I_{i j}, \\
y_{i j}=f\left(x_{i j}\right)=\frac{1}{2}\left(\left|x_{i j}+1\right|-\left|x_{i j}-1\right|\right), \quad i=1, \ldots, m, j=1, \ldots, n,
\end{gathered}
$$

where $I, u, y$, and $x$ denotes input bias, input, output, and state variable of each cell, respectively. $N_{r}(i j)$ is the $t$-neighbourhood of cell $C(k, l)$ as $N_{r}(i j)=\{C(k, l) \mid \max \{|k-i|, \mid l-$ $j \mid\} \leq r\}, i$ and $j$ denote the position of the cell in the network, $k$ and $l$ denote the position 
of the neighbour cell relative to the cell in consideration. $B$ is the nonlinear weights template matrices for input feedback and $A$ is the corresponding template matrices for the outputs of neighbour cells. Non-linearity means that templates can change over time.

A large number of cellular neural networks (CNNs) models have appeared in the literature [2-4], and these models differ in cell complexity, parameterization, cell dynamics, and network topology. Various generalizations of cellular neural networks have attracted attention of scientific community due to their promising potential for tasks of classification, associative memory, parallel computation [5-9], pattern recognition, computer vision, and solving any optimization problem [10-13]. Such applications rely on the existence of equilibrium points and the qualitative properties of cellular neural networks.

Discrete-time cellular neural networks (DT-CNNs) have been studied both in theory and applications. Previous results introduced many properties of DT-CNN in the two dimensional plane. For instance, [14] has been successfully applied to investigate the discretetime analogues of cellular neural network $(\mathrm{CNN})$ with variable coefficients in the twodimensional plane. However, three-dimensional structure is more accurate, specific, and closer to real structures of CNN. Based on the above discussion, this paper proposes some effective results of DT-CNN in the three-dimensional space.

Motivated by the constructing of continuous system (1.1), the discrete analogue of the system (1.1) is considered as follows:

$$
X_{i j}[n+1]=e^{-h} X_{i j}[n]+\left(1-e^{-h}\right)\left(\sum_{C(k, l) \in N r(i j)} A_{k l} Y_{k l}[n]+\sum_{C(k, l) \in N r(i j)} B_{k l} U_{k l}+I_{i j}\right), \quad \forall n \in Z_{0}^{+}
$$

For any $h>0$, the discrete-time analogues (1.2) converge to the continuous-time system (1.1) will be provided. Without loss of generality, (1.2) can be substituted in the DTCNNs model:

$$
\begin{aligned}
& x_{i j}[n+1]=e^{-h} x_{i j}[n]+\left(1-e^{-h}\right) \sum_{C\left(k_{i j h} l_{i j h}\right) \in N r\left(k_{i j h} h_{i j h}\right)}\left(A_{k_{i j h} l_{i j h}} y_{k_{i j h} l_{i j h}}[n]+B_{k_{i j h} l_{i j h}} u_{k_{i j h} h_{i j h}}\right) \\
& +I_{i j}[n], \quad \forall n \in Z_{0}^{+}, \\
& x_{i j}[n]=\varphi_{i j}[n], \quad \forall n \in Z_{0}^{-}=\{0,-1,-2, \ldots\} .
\end{aligned}
$$

Then, the spatial structure with respect to (1.3) is shown in Figure 1, where $r=$ $\max _{C\left(k_{i j h} l_{i j h}\right) \in N_{r}(i j h)}\left(\left|x_{k_{i j h} h_{i j h}}-\partial \Omega\right|\right), \Omega \triangleq\left\{x \in \mathcal{X} \subset N_{r}(i j h),\|x\|<\Theta\right\}, N_{r}(i j h)$ is the $r-$ neighbourhood of a cell $C(k, l)=C_{k_{i j h} l_{i j h}}$, and $\Theta$ will be denoted by the proof of Theorem 3.1 in Section 3.

The rest of the paper is organized as follows: in Section 2, system description and preliminaries are developed in detail and some definitions, assumptions, and lemmas are stated. Section 3 gives sufficient conditions for a periodic solution for DT-CNN in threedimensional space by utilizing continuation theorem of coincidence degree theory. Section 4 proposes global stability of a periodic solution for the DT-CNN. A numerical simulation is given to show correctness of our analysis in Section 5 and concluded in Section 6. 


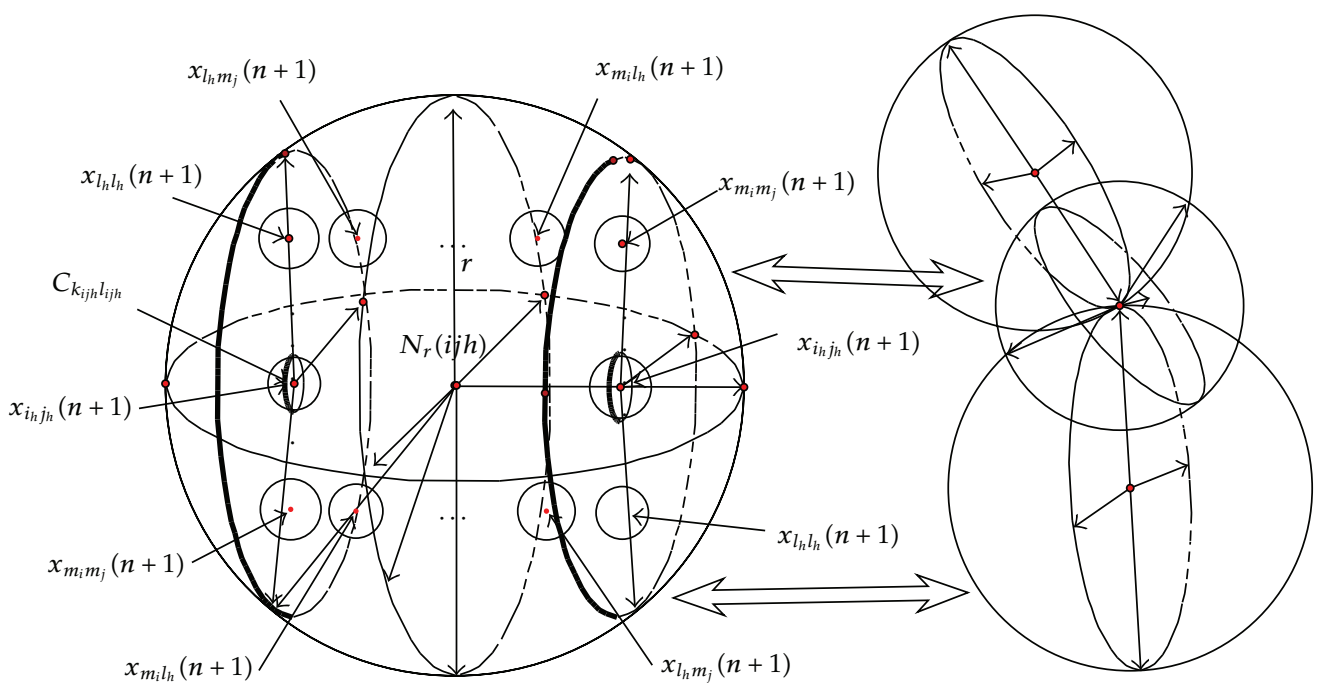

(a)

(b)

Figure 1: Spatial structure with respect to (1.3).

\section{System Preliminaries and Description}

Consider the following model which is equivalent to the (1.3):

$$
\begin{gathered}
x_{i j}[n+1]=\alpha(h) x_{i j}[n]+\beta(h) \sum_{h=1}^{m_{h}} \sum_{j=1}^{m_{j}}\left(A_{k_{i j h} l_{i j h}} y_{k_{i j h} l_{i j h}}[n]+B_{k_{i j h} l_{i j h}} u_{k_{i j h} l_{i j h}}\right)+I_{i j}[n], \quad \forall n \in Z_{0}^{+}, \\
x_{i j}[n]=\varphi_{i j}[n], \quad \forall n \in Z_{0}^{-}=\{0,-1,-2, \ldots\}, \\
k_{i j h}=k(i, j, h) \in N^{+}, \quad l_{i j h}=l(i, j, h) \in N^{+}, \quad i=1,2, \ldots, m_{i}, \\
j=1,2, \ldots, m_{j}, \quad k=1,2, \ldots, m_{k},
\end{gathered}
$$

where $\alpha(h)=e^{-h}, \beta(h)=1-\alpha(h)$, for all $h>0, \varphi_{i j}(n)$ are $N(h)$-periodic sequences, that is, $\varphi_{i j}(n)=\varphi_{i j}(n+N(h))$.

Throughout the paper, the following definitions and lemmas will be introduced.

Definition 2.1 (Fredholm operator). Let $x$ and $y$ be a Banach space, an operator $L$ is called Fredholm operator if $L$ is a bounded linear operator between $\mathcal{x}$ and $y$ whose kernel and cokernel are finite-dimensional and whose range is closed. Equivalently, an operator $L: \mathcal{X} \rightarrow$ $y$ is Fredholm if it is invertible modulo compact operator, that is, if there exists a bounded linear operator $S: y \rightarrow x$ such that $I d_{x}-S L, I d_{y}-L S$ are compact operators on $x$ and $y$, respectively, where $I d_{x}$ and $I d_{y}$ are the identity operator. 
Definition 2.2 ( $L$-compact). An operator $N$ will be called $L$-compact on $\bar{\Omega}$ if the open bounded set $Q N(\bar{\Omega})$ is bounded and $K_{p}(I-Q) N: \bar{\Omega} \rightarrow \mathcal{X}$ is compact, where $K_{p}$ is the inverse operator of $N$. Since $\operatorname{Im} Q$ is isomorphic to $\operatorname{Ker} L$, there exists an isomorphism $J: \operatorname{Im} Q \rightarrow \operatorname{Ker} L$.

The index of a Fredholm operator is ind $L=\operatorname{dim} \operatorname{Ker} L$-codim $\operatorname{Im} L$, then operator $L$ will be called a Fredholm operator of index zero if $\operatorname{dim} \operatorname{Ker} L=\operatorname{codim} \operatorname{Im} L<+\infty$ and $\operatorname{Im} L$ is closed in $\mathcal{Y}$. Then a following abstract equation in Banach space $\mathcal{X}$ is defined by

$$
L x=\lambda N x
$$

Let $L: \operatorname{Dom} L \subset \mathcal{X} \rightarrow \mathcal{y}$ be linear operator, and $N: \mathcal{X} \rightarrow \mathcal{y}$ be a continuous operator. If $L$ is a Fredholm operator of index zero, there must exist continuous projectors $P: \mathcal{X} \rightarrow \mathcal{X}$ and $Q: y \rightarrow y$, such that:

$$
\begin{gathered}
P: \boldsymbol{x} \cap \operatorname{Dom} L \longrightarrow \operatorname{Ker} L, \quad \operatorname{Ker} L=\operatorname{Im} P, \\
Q: y \longrightarrow \boldsymbol{y} / \operatorname{Im} L, \quad \operatorname{Im} L=\operatorname{Ker} Q .
\end{gathered}
$$

In other words, $\left.L\right|_{\operatorname{Dom} L \cap K e r P}: \operatorname{Dom} L \cap \operatorname{Ker} P \rightarrow \operatorname{Im} L$ is invertible, and the inverse of the operator $L$ is denoted by $K_{p}$.

Lemma 2.3 (Gaines and Mawhin [15]). Let $\mathcal{X}$ be a Banach space, $L$ be a Fredholm operator of index zero, and let $N: \bar{\Omega} \rightarrow \mathcal{X}$ be L-compact on $\bar{\Omega}, \Omega \subset \mathcal{X}$, where $\Omega$ is an open bounded set, suppose:

(i) $L x \neq \lambda N x$, for any $(x, \lambda) \in(\partial \Omega \cap \operatorname{Dom} L) \times(0,1)$;

(ii) $Q N x \neq 0$, for any $x \in \partial \Omega \cap \operatorname{Ker} L$;

(iii) $\operatorname{deg}(J Q N, \Omega \cap \operatorname{Ker} L, 0) \neq 0$.

Then $L x=N x$ has at least one solution in Dom $L \cap \bar{\Omega}$.

Lemma 2.4. If $a$ and $b$ are some certain nonnegative vectors, then there exists a positive constant $\beta$, such that $a b \leq(\beta / 2) a^{2}+(1 / 2 \beta) b^{2}$.

Proof. Assuming $a$ and $b$ are some certain non-negative vectors, $\beta$ is a positive constant, then

$$
2 a b=2 a \sqrt{\beta}(\sqrt{\beta})^{-1} b \leq \beta a^{2}+\frac{1}{\beta} b^{2} \Longrightarrow a b \leq \frac{\beta}{2} a^{2}+\frac{1}{2 \beta} b^{2}
$$

Thus, the proof of Lemma 2.4 is completed.

Assumption 2.5. $A_{k_{i j h} l_{j h}}, B_{k_{i j h} l_{i j h}}, I_{i j}\left(i=1, \ldots, m_{i}, j=1, \ldots, m_{j}, h=1, \ldots, m_{h}\right)$ are $N$ periodic sequence of $Z_{0}^{+}$. For the sake of convenience, we use the following notations: 
$\|x\|_{\mathbb{R}^{2}}^{2}=\sum_{j=1}^{m_{j}} \sum_{i=1}^{m_{i}} \max _{n \in I_{N}}\left|x_{i j}[n]\right|^{2}$. For each operator $P: N_{r}(i j h) \rightarrow N_{r}(i j h)$ and any $s=s(u, v, w), t=t(i, j, h) \in N_{r}(i j h)$, such that:

$$
\begin{aligned}
|P(s)-P(t)| & \leq\left(\left|m_{i}\right||u-i|^{2}+\left|m_{j}\right||v-j|^{2}+\left|m_{h}\right||w-h|^{2}\right)^{1 / 2} \\
& \leq 2 m_{i j h} \times \max \left\{\operatorname{dist}\left(s, o_{i j h}\right), \operatorname{dist}\left(t, o_{i j h}\right)\right\} \leq 2 m_{i j h} r
\end{aligned}
$$

where $o_{i j h}$ is the spherical centre of $N_{r}(i j h)$ with a radius length $r, m_{i j h}=\max \left\{\left|m_{i}\right|,\left|m_{j}\right|,\left|m_{h}\right|\right\}$, then it is easy to obtain: $|P(s)-P(t)| \leq\|P\||(s-t)| \leq 2 m_{i j h} r<+\infty$.

Assumption 2.6. There is a positive constant $C_{y_{k l}}$, such that $\left|y_{k_{i j h} l_{i j h}}\left[x_{1}\right]-y_{k_{i j h}} l_{j h}\left[x_{2}\right]\right| \leq C_{y_{k l}} \mid x_{1}-$ $x_{2} \mid$, for all $x_{1} \neq x_{2} \in R$.

\section{Existence of a Periodic Solution with respect to (2.1)}

In many cases, many proposed results are not ideal and therefore it is necessary to formulate a novel and effective result for DT-CNN in the three-dimensional space. Can we obtain the result about the existence and stability of a periodic solution for DT-CNN in threedimensional space? This is the topic we wish to address in this paper. The aim of the present work is to develop a strategy to determine the existence and global stability of a periodic solution with respect to (2.1) in the three-dimensional space. Consequently, we processed with the following result.

Theorem 3.1. Suppose that Assumptions 2.5 and 2.6 hold, and the following condition holds:

$$
\sum_{h=1}^{m_{h}}\left|\widetilde{B}_{k_{i j h} l_{i j h}} u_{k_{i j h} l_{j i h}}\right|^{2}-\gamma_{i j}>0
$$

where $\gamma_{i j}=\sum_{h=1}^{m_{h}}\left|\tilde{A}_{k_{i j h} l_{i j h}} y_{k_{i j h} l_{i j h}}\right|^{2}-\widetilde{I}_{i j}^{2}, i=1, \ldots, m_{i}, j=1, \ldots, m_{j}, n \in I_{N}=\{0,1, \ldots, N-1\}$, then (2.1) has at least one $N$-periodic solution.

Proof. In this section, by means of using Mawhin's continuation theorem of coincidence degree theory, we will study the existence of at least one periodic solution with respect to (2.1), for convenience, some following notations will be used:

$$
I_{N}=\{0,1, \ldots, N-1\}, \quad \underline{f}=\min _{n \in I_{N}}\{|f(n)|\}, \quad \bar{f}=\max _{n \in I_{N}}\{|f(n)|\},
$$

where $f(n)$ is any function. Let $\mathcal{X}=\boldsymbol{y}=x[n]=\left\{\left(x_{11}[n], \ldots, x_{1 m_{j}}[n], \ldots, x_{m_{i} 1}[n], \ldots\right.\right.$, $\left.\left.x_{m_{i} m_{j}}[n]\right)^{T}: x_{i j}[n]=x_{i j}[n+N] \in R_{+}^{m_{i} m_{j}}, N \in N^{+}, i=1, \ldots, m_{i}, j=1, \ldots, m_{j}\right\}$, and $y^{N} \subset x=y$ be the subspace of all $N$-periodic sequence; equip it with the norm $\|x\|_{\mathbb{R}^{2}}^{2}=$ $\sum_{j=1}^{m_{j}} \sum_{i=1}^{m_{i}} \max _{n \in I_{N}}\left|x_{i j}[n]\right|^{2}$. For any $\varepsilon>0,\left\{x_{i_{m}}\right\}_{i_{m}=1}^{m_{i}} \subset N_{r}(i j h)$, there exists $N(\varepsilon)>0$ and $\varepsilon>0$, such that $i_{m}>N(\varepsilon) \Rightarrow d\left(x_{i_{m}}, x_{i_{m}+1}\right) \leq d\left(x_{i_{m}}, o_{i j h}\right)+d\left(x_{i_{m}+1}, o_{i j h}\right)<\varepsilon$. Thus, $\left\{x_{i_{m}}\right\}_{i_{m}=1}^{m_{i}}$ is a Cauchy sequence in $N_{r}(i j h)$ and $o_{i j h}$ is the spherical centre of $N_{r}(i j h), d(x, y)=\max \{|x-y|$ : $x \in X\}$. By utilizing the meaning of $N_{r}(i j h)$ and Bolzano-Weierstrass theorem (Each bounded sequence in $R^{n}$ has a convergent subsequence, here $\left.R^{m_{i} m_{j}} \subset R^{n}, \operatorname{dim}\left(R^{m_{i} m_{j}}\right)<+\infty\right)$, it is easy to know that $(x,\|\cdot\|)$ is a Banach space. 
Set

$$
\begin{gathered}
N x=\left(\widehat{x}_{11}[n], \ldots, \widehat{x}_{1 m_{j}}[n], \widehat{x}_{21}[n], \ldots, \widehat{x}_{2 m_{j}}[n], \widehat{x}_{m_{i} 1}[n], \ldots, \widehat{x}_{m_{i} m_{j}}[n]\right)^{T}, \\
L x=\left(\Delta x_{11}[n], \ldots, \Delta x_{1 m_{j}}[n], \Delta x_{21}[n], \ldots, \Delta x_{2 m_{j}}[n], \Delta x_{m_{i}}[n], \ldots, \Delta x_{m_{i} m_{j}}[n]\right)^{T}, \\
P x=Q x=\left(\tilde{x}_{11}[n], \ldots, \tilde{x}_{1 m_{j}}[n], \tilde{x}_{21}[n], \ldots, \tilde{x}_{2 m_{j}}[n], \tilde{x}_{m_{i} 1}[n], \ldots, \tilde{x}_{m_{i} m_{j}}[n]\right)^{T},
\end{gathered}
$$

that is

$$
\begin{gathered}
x[n]=\left(x_{11}[n], \ldots, x_{1 m_{j}}[n], \ldots, x_{m_{i}}[n], \ldots, x_{m_{i} m_{j}}[n]\right)^{T} \in \mathcal{X}, \\
\operatorname{Ker} L=\left\{x=\{x[n]\} \in y^{N} \subset x: x[n]=c \in R^{m_{i} m_{j}}, n \in I_{N}\right\}, \\
\operatorname{Im} L=\left\{x=\{x[n]\} \in y^{N} \subset x: \sum_{n=0}^{N-1} x_{i j}[n]=0, i=1, \ldots, m_{i}, j=1, \ldots, m_{j}, n \in I_{N}\right\},
\end{gathered}
$$

where $\Delta x_{i j}[n]=x_{i j}[n+1]-x_{i j}[n]=-\beta(h)\left[x_{i j}[n]-\sum_{h=1}^{m_{h}} \sum_{j=1}^{m_{j}}\left(A_{k_{i j h} l_{i j h}} y_{k_{i j h} l_{j h h}}[n]+B_{k_{i j h} l_{i j h}} u_{k_{i j h} l_{j h}}\right)\right]+$ $I_{i j}[n], \widehat{x}_{i j}[n]=-\beta(h)\left[x_{i j}[n]-\sum_{h=1}^{m_{h}} \sum_{j=1}^{m_{j}}\left(A_{k_{i j h} h_{i j h}} y_{k_{i j h} l_{i j h}}[n]+B_{k_{i j h} l_{i j h}} u_{k_{i j h} l_{i j h}}\right)\right]+I_{i j}[n], \tilde{x}=$ $(1 / N) \sum_{n=0}^{N-1} x[n]$, for all $n \in I_{N}$. Then we will learn that $\operatorname{dim} \operatorname{Ker} L=\operatorname{codim} \operatorname{Im} L<+\infty$, it is easy to prove that $L$ is a bounded linear operator, $P$ and $Q$ are two continuous operators such that $\operatorname{Ker} L=\operatorname{Im} P, \operatorname{Im} L=\operatorname{Ker} Q=\operatorname{Im}(I-Q)$, and $\left.K_{p}\right|_{\operatorname{Im} L}: \operatorname{Im} L \rightarrow \operatorname{Dom} L \cap \operatorname{Ker} P$, that is

$$
K_{p}(x[n])=\sum_{s=0}^{N-1} x(s)-\frac{1}{N} \sum_{s=1}^{N} \sum_{t=1}^{s-1} x(t), \quad n \in I_{N}
$$

that is

$$
\begin{aligned}
& Q N x \\
& =\frac{\beta(h)}{1+\beta(h)}
\end{aligned}
$$

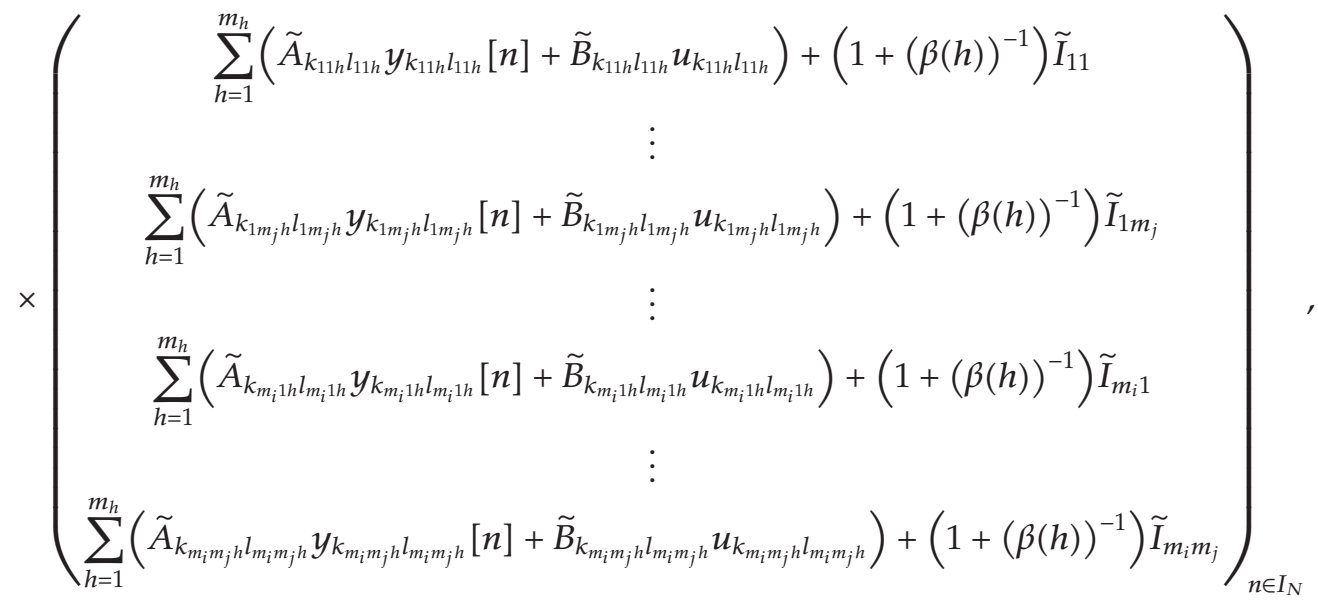




$$
\begin{aligned}
& K_{p}(I-Q) N x \\
& =-\beta(h)
\end{aligned}
$$

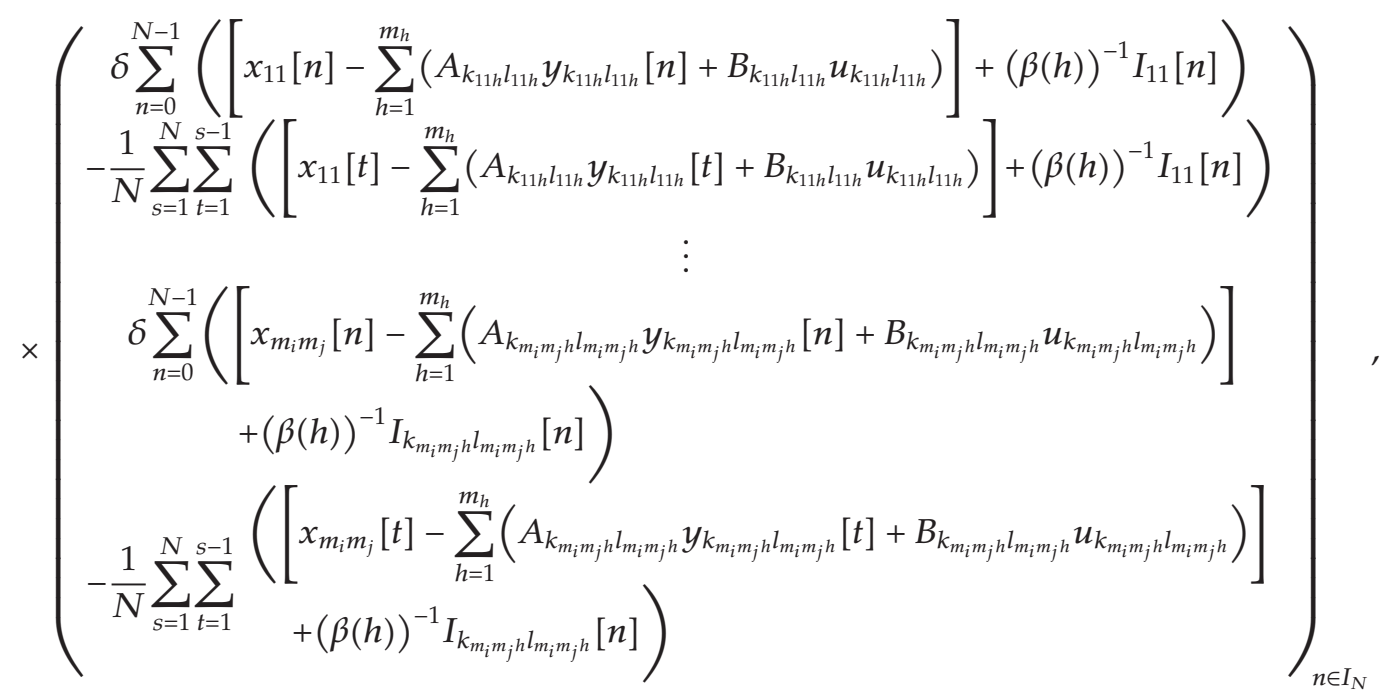

where $\delta(\beta(h), n, N)$ is a constant, which is only depended on variables $h, n$, and $N$.

Obviously, employing the Lebesgue's convergence theorem, we can easily learn that $Q N(\bar{\Omega})$ is bounded, $K_{p}(I-Q) N(\bar{\Omega})$ is compact for any open bounded set $\Omega \subset \mathcal{X} \subset N_{r}(i j h)$ by using Ascoli-Arzela's theorem (A subset $\mathcal{F}$ of $\mathcal{C}(\mathcal{X})$ is compact if and only if it is closed, bounded and equi-continuous). Thus, $N$ is $L$-compact on a closed set $\bar{\Omega}$ with any open bounded set $\Omega \subset \mathcal{X} \subset N_{r}(i j h)$.

Suppose that $x[n]=\left(x_{11}[n], \ldots, x_{1 m_{j}}[n], \ldots, x_{m_{i} 1}[n], \ldots, x_{m_{i} m_{j}}[n]\right)^{T} \in \mathcal{X}$ is a solution with respect to $(2.1)$, for certain $\lambda \in(0,1)$. Then the following equation can be derived by (2.2):

$$
\begin{aligned}
\Delta x_{i j}[n] & =x_{i j}[n+1]-x_{i j}[n] \\
& =-\lambda\left\{\beta(h)\left[x_{i j}[n]-\sum_{h=1}^{m_{h}} \sum_{j=1}^{m_{j}}\left(A_{k_{i j h} l_{i j h}} y_{k_{i j h} l_{i j h}}[n]+B_{k_{i j h} l_{i j h}} u_{k_{i j h} h_{i j h}}\right)\right]+I_{i j}[n]\right\}, \quad \forall n \in I_{N} .
\end{aligned}
$$

Then, the following results can be derived by utilizing (3.7):

$$
\begin{aligned}
& \max _{n \in I_{N}}\left|x_{i j}[n]\right|=\max _{n \in I_{N}} \mid x_{i j}[n+1] \mid \\
& \leq \max _{n \in I_{N}}\left[(1-\lambda \beta(h))\left|x_{i j}[n]\right|+\lambda \beta(h)\right. \\
&\left.\quad \times \sum_{h=1}^{m_{h}} \sum_{j=1}^{m_{j}}\left(\left|A_{k_{i j h} l_{i j h}} y_{k_{i j h} l_{i j h}}[n]\right|+\left|B_{k_{i j h} l_{i j h}} u_{k_{i j h} l_{i j h}}\right|\right)+\left|\lambda I_{i j}[n]\right|\right]
\end{aligned}
$$




$$
\begin{aligned}
\leq & \max _{n \in I_{N}}(1-\lambda \beta(h))^{n}\left|\varphi_{i j}[0]\right|+(\lambda(1-\alpha(h)))^{n} \\
& \times \sum_{h=1}^{m_{h}} \sum_{j=1}^{m_{j}}\left(\left|\bar{A}_{k_{i j h} l_{i j h}} y_{k_{i j h} l_{i j h}}[0]\right|\right)+\lambda^{n} \bar{I}_{i j}+\sigma_{i j} \\
\leq & \max _{n \in I_{N}}\left\{\left|\varphi_{i j}[0]\right|+(1-\alpha(h))^{n} \sum_{h=1}^{m_{h}} \sum_{j=1}^{m_{j}} \bar{A}_{k_{i j h} l_{i j h}}\right\}+\sigma_{i j}<+\infty,
\end{aligned}
$$

where $\sigma_{i j}=\max _{n \in I_{N}}(1-\alpha(h)) \sum_{h=1}^{m_{h}} \sum_{j=1}^{m_{j}}\left|\bar{B}_{k_{i j h} l_{i j h}} \bar{u}_{i j h} l_{i j h}\right|+\bar{I}_{i j}$, for all $n \in I_{N}$. Therefore, the solution with respect to $(2.1)$ is bounded for certain $\lambda \in(0,1)$. In other words,

$$
\begin{aligned}
\max _{n \in I_{N}}\left|x_{i j}[n]\right| \leq \max _{n \in I_{N}}\left\{\left|\varphi_{i j}[0]\right|+(1-\alpha(h))^{n} \sum_{h=1}^{m_{h}} \sum_{j=1}^{m_{j}}\left(\left|\bar{A}_{k_{i j h} l_{i j h}} y_{k_{i j h} h_{i j h}}[0]\right|\right)\right. \\
\left.+(1-\alpha(h)) \sum_{h=1}^{m_{h}} \sum_{j=1}^{m_{j}}\left|\bar{B}_{k_{i j h} l_{i j h}} \bar{u}_{k_{i j h} l_{i j h}}\right|+\bar{I}_{i j}\right\} \triangleq \Theta_{i j} .
\end{aligned}
$$

Then the open bounded set $\Omega$ is presented as follows:

$$
\Omega \triangleq\left\{x \in \mathcal{X} \subset N_{r}(i j h),\|x\|<\sum_{i=1}^{m_{i}} \sum_{j=1}^{m_{j}} \Theta_{i j}\right\} .
$$

Thus $L x \neq \lambda N x$ for any $(x, \lambda) \in(\partial \Omega \cap \operatorname{Dom} L) \times(0,1)$, the $\Omega$ satisfies condition (i) in Lemma 2.3.

In Figure 2, the nonlinear weights template matrices $B$ and the boundary of $\Omega$ are shown, respectively. Then for any two dimensional plane of any spherical neighbourhood is denoted. Thus, for any $x \in \partial \Omega \cap \operatorname{Ker} L=\partial \Omega \cap R^{m_{i} m_{j}}, \operatorname{Ker} L=\left\{x=\{x[n]\} \in y^{N} \subset \mathcal{X}: x[n]=\right.$ $\left.c \in R^{m_{i} m_{j}}, n \in I_{N}\right\}$, it is easy to learn that $x$ is a constant vector in $R^{m_{i} m_{j}}$ with $\|x\|=\Theta$; Thus, we have

$$
Q N x=\left(Q N x_{11}, \ldots, Q N x_{1 m_{j}}, \ldots, Q N x_{m_{i} 1}, \ldots, Q N x_{m_{i} m_{j}}\right)^{T}
$$

where $Q N x_{i j}[n]=(\beta(h) /(1+\beta(h))) \sum_{h=1}^{m_{h}} \sum_{j=1}^{m_{j}}\left(\tilde{A}_{k_{i j h} l_{i j h}} y_{k_{i j h} l_{i j h}}[n]+\widetilde{B}_{k_{i j h} l_{i j h}} u_{k_{i j h} l_{i j h}}\right)+\widetilde{I}_{i j}[n]$, for all $n \in I_{N}$. Furthermore, we can calculate the bound of $Q N x$ as follows:

$$
\begin{aligned}
\|Q N x\|^{2} & =\sum_{j=1}^{m_{j}} \sum_{i=1}^{m_{i}} \max _{n \in I_{N}}\left|x_{i j}[n]\right|^{2} \\
& =\sum_{j=1}^{m_{j}} \sum_{i=1}^{m_{i}} \max _{n \in I_{N}}\left|\frac{\beta(h)}{1+\beta(h)} \sum_{h=1}^{m_{h}} \sum_{j=1}^{m_{j}}\left(\widetilde{A}_{k_{i j h} l_{i j h}} y_{k_{i j h} l_{i j h}}[n]+\widetilde{B}_{k_{i j h} l_{j j h}} u_{k_{i j h} l_{i j h}}\right)+\widetilde{I}_{i j}[n]\right|^{2}
\end{aligned}
$$


Discrete Dynamics in Nature and Society

$$
\begin{aligned}
& \geq \sum_{j=1}^{m_{j}} \sum_{i=1}^{m_{i}}\left|\frac{\beta(h)}{1+\beta(h)}\right|^{2} \times \max _{n \in I_{N}}\left\{\sum_{h=1}^{m_{h}}\left|\widetilde{B}_{k_{i j h} l_{i j h}} u_{k_{i j h} l_{j h h}}\right|^{2}+\widetilde{I}_{i j}^{2}[n]-\sum_{h=1}^{m_{h}}\left|\tilde{A}_{k_{i j h} l_{i j h}} y_{k_{i j h} l_{i j h}}\right|^{2}\right\} \\
& \geq \sum_{j=1}^{m_{j}} \sum_{i=1}^{m_{i}}\left(\max _{n \in I_{N}}\left(\sum_{h=1}^{m_{h}}\left|\widetilde{B}_{k_{i j h} l_{i j h}} u_{k_{i j h} h_{i j h}}\right|^{2}-\gamma_{i j}\right)\right)>0,
\end{aligned}
$$

where $\gamma_{i j}=\sum_{h=1}^{m_{h}}\left|\widetilde{A}_{k_{i j h} l_{i j h}} y_{k_{i h h} h_{i j h}}\right|^{2}-\widetilde{I}_{i j}^{2}, i=1, \ldots, m_{i}, j=1, \ldots, m_{j}, n \in I_{N}$. Thus for any $x=$ $\partial \Omega \cap \operatorname{Ker} L, Q N x \neq 0$, this proves the condition (ii) in Lemma 2.3.

In order to prove the condition (iii) is satisfied with respect to (2.1), we only need to prove that $\operatorname{deg}(J Q N, \Omega \cap \operatorname{Ker} L, 0) \neq 0$. Define $\Phi: \operatorname{Dom} L \rightarrow \chi x$ by

$$
\Phi\left(x_{11}, \ldots, x_{1 m_{j}}, \ldots, x_{m_{i} 1}, \ldots, x_{m_{i} m_{j}}\right)^{T}=\left(\tilde{x}_{11}, \ldots, \tilde{x}_{1 m_{j}}, \ldots, \tilde{x}_{m_{i}} 1, \ldots, \tilde{x}_{m_{i} m_{j}}\right)^{T}
$$

where $\tilde{x}_{i j}[n]=(\beta(h) /(1+\beta(h))) \sum_{h=1}^{m_{h}} \sum_{j=1}^{m_{j}}\left(\tilde{A}_{k_{i j h} l_{j h}} y_{k_{i j h} l_{i j h}}[n]+\widetilde{B}_{k_{i j h} l_{i j h}} u_{k_{i j h} h_{i j h}}\right)+\tilde{I}_{i j}[n]$, for all $n \in I_{N}$.

Now we will prove that $x \in \partial \Omega \cap \operatorname{Ker} L=\partial \Omega \cap R^{m_{i} m_{j}}, \Phi\left(x_{11}, \ldots, x_{1 m_{j}}, \ldots, x_{m_{i}} 1\right.$, $\left.\ldots, x_{m_{i} m_{j}}\right)^{T} \neq(0,0, \ldots, 0)^{T}$. If this is not true, then $x \in \partial \Omega \cap \operatorname{Ker} L=\partial \Omega \cap R^{m_{i} m_{j}}, \Phi\left(x_{11}\right.$, $\left.\ldots, x_{1 m_{j}}, \ldots, x_{m_{i}}, \ldots, x_{m_{i} m_{j}}\right)^{T}=(0,0, \ldots, 0)^{T}$, thus, for constant vector $x \in \partial \Omega$, we have:

$$
\tilde{x}_{i j}[n]=\frac{\beta(h)}{1+\beta(h)} \sum_{h=1}^{m_{h}} \sum_{j=1}^{m_{j}}\left(\tilde{A}_{k_{i j h} l_{i j h}} y_{k_{i j h} l_{i j h}}[n]+\widetilde{B}_{k_{i j h} l_{i j h}} u_{k_{i j h} l_{i j h}}\right)+\tilde{I}_{i j}[n]=0
$$

Equivalently, (3.14) can be written as the following form:

$$
\begin{aligned}
& \sum_{h=1}^{m_{h}} \sum_{j=1}^{m_{j}}\left(\widetilde{A}_{k_{i j h} l_{i j h}} y_{k_{i j h} l_{i j h}}[n]+\widetilde{B}_{k_{i j h} l_{i j h}} u_{k_{i j h} l_{j i h}}\right) \\
& \quad=-\frac{1+\beta(h)}{\beta(h)} \widetilde{I}_{i j}[n] \\
& \quad \Longrightarrow \sum_{h=1}^{m_{h}} \sum_{j=1}^{m_{j}} \widetilde{A}_{k_{i j h} l_{i j h}} y_{k_{i j h} l_{i j h}}[n]=-\left(\frac{1+\beta(h)}{\beta(h)} \widetilde{I}_{i j}[n]+\sum_{h=1}^{m_{h}} \sum_{j=1}^{m_{j}} \widetilde{B}_{k_{i j h} l_{i j h}} u_{k_{i j h} l_{i j h}}\right) .
\end{aligned}
$$

Combining (3.12) and (3.15), the following results are obtained:

$$
\max _{n \in I_{N}} \sum_{h=1}^{m_{h}} \sum_{j=1}^{m_{j}}\left|\widetilde{A}_{k_{i j h} l_{i j h}} y_{k_{i j h} l_{i j h}}\right|=\max _{n \in I_{N}}\left|\frac{1+\beta(h)}{\beta(h)} \widetilde{I}_{i j}+\sum_{h=1}^{m_{h}} \sum_{j=1}^{m_{j}} \widetilde{B}_{k_{i j h} l_{i j h}} u_{k_{i j h} l_{i j h}}\right| .
$$




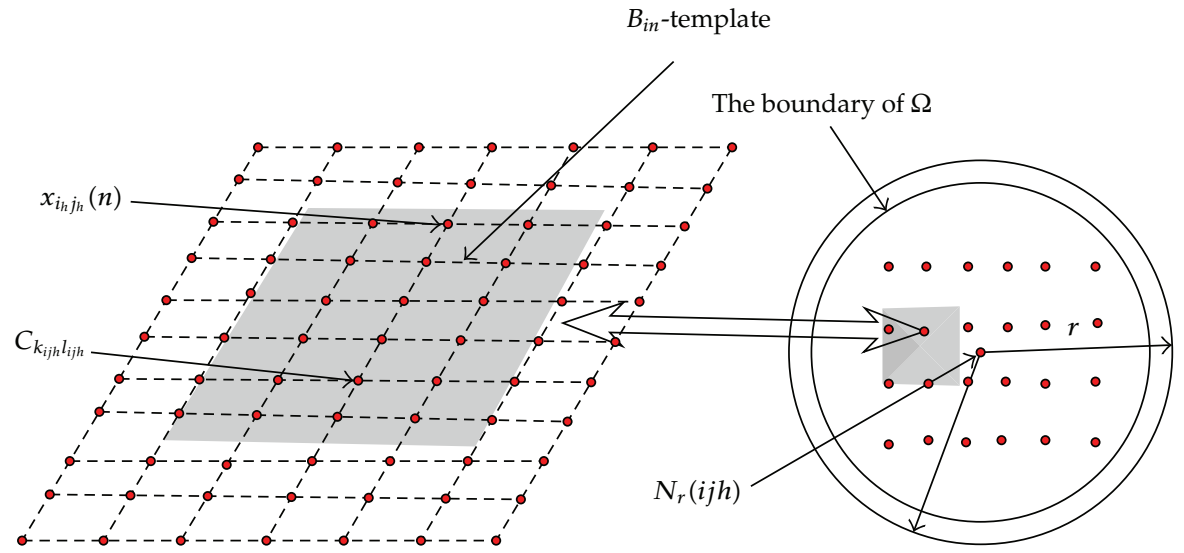

Figure 2: Input $B$-template and the boundary of $\Omega$.

Thus, the following result is derived by calculating the (3.16):

$$
\begin{aligned}
& \left|\frac{1+\beta(h)}{\beta(h)} \widetilde{I}_{i j}+\sum_{h=1}^{m_{h}} \sum_{j=1}^{m_{j}} \widetilde{B}_{k_{i j h} l_{i j h}} u_{k_{i j h} l_{i j h}}\right|-\sum_{h=1}^{m_{h}} \sum_{j=1}^{m_{j}}\left|\widetilde{B}_{k_{i j h} l_{i j h}} u_{k_{i j h} l_{i j h}}\right| \\
& \quad \leq\left|\frac{1+\beta(h)}{\beta(h)} \widetilde{I}_{i j}\right|<\left|\widetilde{I}_{i j}\right| \\
& \quad<\max _{n \in I_{N}}\left\{\sum_{h=1}^{m_{h}} \sum_{j=1}^{m_{j}}\left|\widetilde{B}_{k_{i j h} l_{i j h}} u_{k_{i j h} l_{i j h}}\right|+\widetilde{I}_{i j}\right\} .
\end{aligned}
$$

Obviously, (3.17) is a contradiction since $((1+\beta(h)) / \beta(h))>1$, then for any $x=$ $\partial \Omega \cap \operatorname{Ker} L, \operatorname{Ker} L=\operatorname{Im} P, P x=\left(\tilde{x}_{11}[n], \ldots, \tilde{x}_{1 m_{j}}[n], \tilde{x}_{m_{i} 1}[n], \ldots, \tilde{x}_{m_{i} m_{j}}[n]\right)^{T} \neq(0, \ldots, 0)^{T}$, $\Phi\left(x_{11}, \ldots, x_{1 m_{j}}, \ldots, x_{m_{i} 1}, \ldots, x_{m_{i} m_{j}}\right)^{T} \neq(0, \ldots, 0)^{T}$. Thus, $\operatorname{deg}(J Q N, \Omega \cap K e r L, 0) \neq 0$. Therefore, (2.1) has at least one $N$-periodic solution, thus the proof of Theorem 3.1 is completed.

Corollary 3.2. Suppose that Assumptions 2.5 and 2.6 hold, and the following condition holds:

$$
\sum_{h=1}^{m_{h}}\left|\tilde{A}_{k_{i j h} l_{i j h}} y_{k_{i j h} l_{i j h}}\right|^{2}-\eta_{i j}>0,
$$

where $\eta_{i j}=\sum_{h=1}^{m_{h}}\left|\widetilde{B}_{k_{i j h} l_{i j h}} u_{k_{i j h} h_{i j h}}\right|^{2}-\widetilde{I}_{i j^{\prime}}^{2} i=1, \ldots, m_{i}, j=1, \ldots, m_{j}, n \in I_{N}$, then (2.1) has at least one N-periodic solution.

Proof. Similar to the proof of Theorem 3.1, so it is omitted. 


\section{Globally Stability of a Periodic Solution with respect to (2.1)}

The existence of a periodic solution for the system (2.1) is derived in the Theorem 3.1. Then global stability of a periodic solution with respect to (2.1) in the three-dimensional space is presented in the following.

Theorem 4.1. Suppose that Assumptions 2.5 and 2.6 hold, and the following condition holds:

$$
\sum_{h=1}^{m_{h}} \sum_{j=1}^{m_{j}} \bar{A}_{k_{i j h} l_{i j h}} \leq \frac{1}{C_{y_{k l}} m_{h} m_{j}}
$$

where $i=1,2, \ldots, m_{i}, C_{y_{k l}}, m_{j}$ and $m_{h}$ are positive constants, then the periodic solution with respect to (2.1) is global stability.

Proof. It follows from the Theorem 3.1 that (2.1) has at least a periodic solution, without loss of generality, the periodic solution can be described by:

$$
x^{*}[n]=\left(x_{11}^{*}[n], \ldots, x_{1 m_{j}}^{*}[n], \ldots, x_{m_{i} 1}^{*}[n], \ldots, x_{m_{i} m_{j}}^{*}[n]\right)^{T} \in \mathcal{X}
$$

Then we can define the following formula:

$$
u_{i j}[n]=\left|x_{i j}[n]-x_{i j}^{*}[n]\right|, \quad i=1, \ldots, m_{i}, j=1, \ldots, m_{j}
$$

Now, we show that the a periodic solution $x^{*}[n]$ is globally stable, and the following inequality is obtained by utilizing (2.1) and (4.3):

$$
\begin{aligned}
u_{i j}[n+1]= & \left|x_{i j}[n+1]-x_{i j}^{*}[n+1]\right| \\
\leq & \left\{\alpha(h)\left|x_{i j}[n]-x_{i j}^{*}[n]\right|+C_{y_{k l} l} \beta(h) \sum_{h=1}^{m_{h}} \sum_{j=1}^{m_{j}} A_{k_{i j h} l_{i j h}}\left|y_{k_{i j h} l_{i j h}}[n]-y_{k_{i j h} l_{i j h}}^{*}[n]\right|\right\} \\
\leq & \left\{\alpha(h)\left[\left|x_{i j}[n]-x_{o_{i j h}}[n]\right|+\left|x_{o_{i j h}}[n]-x_{i j}^{*}[n]\right|\right]+C_{y_{k l}} \beta(h) \sum_{h=1}^{m_{h}} \sum_{j=1}^{m_{j}} A_{k_{i j h} l_{i j h}}\right. \\
& \left.\times\left[\left|x_{k_{i j h} l_{i j h}}[n]-x_{o_{i j h}}[n]\right|+\left|x_{o_{i j h}}[n]-x_{k_{i j h} l_{i j h}}^{*}[n]\right|\right]\right\} \\
\leq & 2 r\left(\alpha(h)+C_{y_{k l}} \beta(h) m_{h} m_{j} \sum_{h=1}^{m_{h}} \sum_{j=1}^{m_{j}} \bar{A}_{k_{i j h} l_{i j h}}\right)<+\infty .
\end{aligned}
$$


We design the following Lyapunov-type sequence $V[n]$ by

$$
V[n]=\sum_{i=1}^{m_{i}} \sum_{j=1}^{m_{j}}\left|x_{i j}[n]-x_{i j}^{*}[n]\right|
$$

Then, we can calculate the $\Delta V[n]$ by combining (2.1) and (4.5):

$$
\begin{aligned}
\Delta V[n] & =V[n+1]-V[n] \\
& =\sum_{i=1}^{m_{i}} \sum_{j=1}^{m_{j}}\left(\left|x_{i j}[n+1]-x_{i j}^{*}[n+1]\right|-\left|x_{i j}[n]-x_{i j}^{*}[n]\right|\right) \\
& \leq \sum_{i=1}^{m_{i}} \sum_{j=1}^{m_{j}}\left\{-\beta(h)\left|x_{i j}[n]-x_{i j}^{*}[n]\right|+C_{y_{k l}} \beta(h) \sum_{h=1}^{m_{h}} \sum_{j=1}^{m_{j}} A_{k_{i j h} l_{i j h}} \mid y_{\left.k_{i j h} l_{i j h}[n]-y_{k_{i j h} l_{i j h}}^{*}[n] \mid\right\}}\right. \\
& \leq \sum_{h=1}^{m_{h}} \sum_{j=1}^{m_{j}} 2 r \beta(h)\left(C_{y_{k l}} m_{h} m_{j} \sum_{h=1}^{m_{h}} \sum_{j=1}^{m_{j}} \bar{A}_{k_{i j h} l_{i j h}}-1\right) \leq 0 .
\end{aligned}
$$

Thus, it is easy to obtain $V[n] \leq V[0]$ by the meaning of the (4.6), and furthermore,

$$
\begin{gathered}
\sum_{i=1}^{m_{i}} \sum_{j=1}^{m_{j}}\left|x_{i j}[n]-x_{i j}^{*}[n]\right| \leq \beta(h)\left\{C_{y_{k l}} m_{h} m_{j} \sum_{h=1}^{m_{h}} \sum_{j=1}^{m_{j}} \bar{A}_{k_{i j h} l_{i j h}}-1\right\} \sup _{s \in Z_{0}^{-}} d[s] \\
\Longrightarrow \sum_{i=1}^{m_{i}} \sum_{j=1}^{m_{j}}\left|x_{i j}[n]-x_{i j}^{*}[n]\right| \leq \beta(h)\left\{C_{y_{k l}} m_{h} m_{j} \sum_{h=1}^{m_{h}} \sum_{j=1}^{m_{j}} \bar{A}_{k_{i j h} l_{i j h}}-1\right\} \sup _{s \in Z_{0}^{-}} d[s] \leq 0 \\
\Longrightarrow x_{i j}[n]=x_{i j}^{*}[n], \quad n \in I_{N}=\{0,1, \ldots, N-1\},
\end{gathered}
$$

where $d[s]=\max _{s \in Z_{0}^{-}}\left\{\left|x_{k_{i j h} l_{i j h}}[s]-x_{k_{i j h} l_{i j h}}^{*}[s]\right|,\left|x_{i j}[s]-x_{i j}^{*}[s]\right|\right\}$. Obviously, from the proof of Theorem 4.1, the globally stable of a periodic solution with respect to (2.1) is derived. Then, existence and global stability of a periodic solution for DT-CNNs are obtained by utilizing the conditions of the proposed theorems in an arbitrary diameter plane of a convex space. Thus the proof of Theorem 4.1 is completed. 


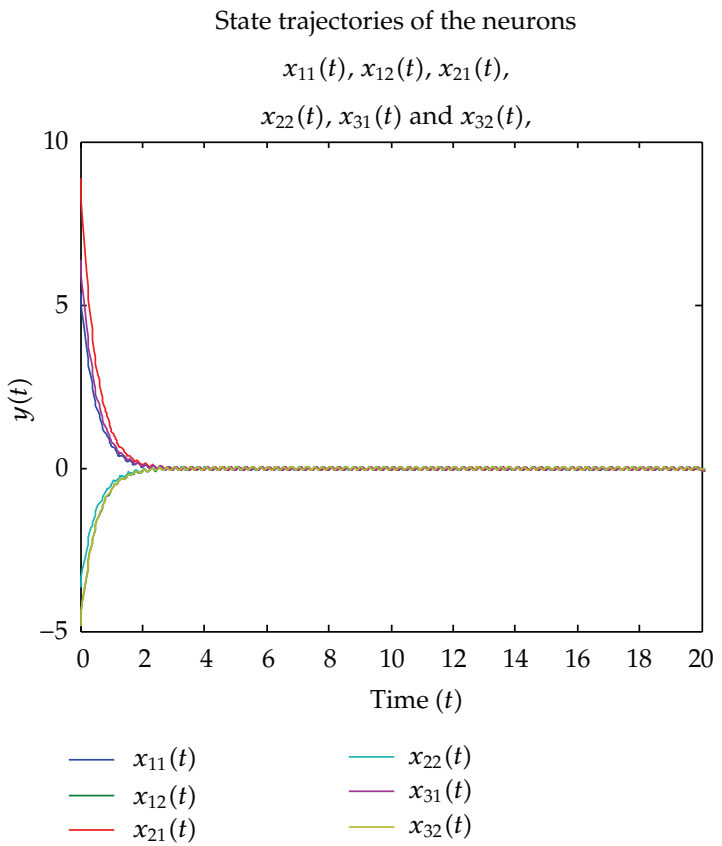

Figure 3: State trajectories of the neurons $x_{11}, x_{12}, x_{21}, x_{22}, x_{31}, x_{32}$.

\section{Numerical Simulation}

In this section, we give an example to show the effectiveness and improvement of the derived results. Consider the following continuous cellular neural networks:

$$
\begin{aligned}
& C \frac{\mathrm{d} x_{i j}}{\mathrm{~d} t}=-\frac{1}{R} x_{i j}+\sum_{C(k, l) \in N_{r}(i j)} A_{k l} y_{k l}+\sum_{C(k, l) \in N_{r}(i j)} B_{k l} u_{k l}+I_{i j}, \\
& y_{i j}=f\left(x_{i j}\right)=\frac{1}{2}\left(\left|x_{i j}+1\right|-\left|x_{i j}-1\right|\right), \quad i=1,2,3, j=1,2,
\end{aligned}
$$

for $t>0$, where $C=1, R=1 / 2, y_{k l}=(1 / 2)\left(\left|x_{i j}+1\right|-\left|x_{i j}-1\right|\right), A_{k_{111} l_{111}}=A_{k_{311} l_{311}}=$ $A_{k_{321} l_{321}}=0.1 \sin (8 \pi t), A_{k_{121} l_{121}}=A_{k_{211} l_{211}}=A_{k_{221} l_{221}}=0.1 \cos (8 \pi t), B_{k_{i 11} l_{i 11}} u_{k_{i 11} l_{i 11}}=-0.06+$ $0.08 \sin (8 \pi t), B_{k_{i 21} l_{i 21}} u_{k_{i 21} l_{i 21}}=-0.02+0.06 \cos (8 \pi t), I_{i 1}=\sin (8 \pi t), I_{i 2}=\cos (8 \pi t), i=$ $1,2,3, x[0]=(5.386,-4.836,8.863,-3.683,6.386,-4.836)^{T}$. Then, state trajectories of $x_{i j}(i=$ $1,2,3, j=1,2)$ are denoted in Figure 3.

From Figure 3, it is easy to know that a $(1 / 4)$-periodic solution of the continuous cellular neural networks is globally stable. Compared to the system (5.1), we design the discrete-time analogue of the continuous cellular neural network as follows:

$$
\begin{aligned}
x_{i j}[n+1]= & \alpha(h) x_{i j}[n]+\beta(h) \sum_{h=1}^{m_{h}} \sum_{j=1}^{m_{j}}\left(A_{k_{i j h} l_{i j h}} y_{k_{i j h} l_{i j h}}[n]+B_{k_{i j h} l_{i j h}} u_{k_{i j h} l_{i j h}}\right) \\
& +I_{i j}[n], \quad n \in Z_{0}^{+}, \quad i=1,2,3, j=1,2,
\end{aligned}
$$




$$
\begin{aligned}
x[0] & =\left(x_{11}[0], x_{12}[0], x_{21}[0], x_{22}[0], x_{31}[0], x_{32}[0]\right)^{T} \\
& =(5.386,-4.836,8.863,-3.683,6.386,-4.836)^{T},
\end{aligned}
$$

for $h>0$, by using Assumptions 2.5 and 2.6 in Section 2, each variable is denoted as:

$$
\begin{aligned}
& \alpha(h)=e^{-h}, \quad \beta(h)=1-e^{-h}, \\
& A_{k_{111} l_{111}}=A_{k_{311} l_{311}}=A_{k_{321} l_{321}}=\frac{1}{20}\left(1-e^{-2 h}\right) \sin (8 \pi n h), \\
& y_{k_{i j h} l_{j h h}}[n]=\frac{1}{2}\left(\left|x_{k_{i j h} l_{i j h}}[n]+1\right|-\left|x_{k_{i j h} l_{i j h}}[n]-1\right|\right), \\
& A_{k_{121} l_{121}}=A_{k_{211} l_{211}}=A_{k_{221} l_{221}}=\frac{1}{20}\left(1-e^{-2 h}\right) \sin (8 \pi n h), \quad I_{i 1}=\frac{1}{20}\left(1-e^{-2 h}\right) \sin (8 \pi n h), \\
& I_{i 2}=\frac{1}{20}\left(1-e^{-2 h}\right) \cos (8 \pi n h), \quad B_{k_{i 11} l_{i 11}} u_{k_{i 11} l_{i 11}}=-0.02+0.08 \sin (8 \pi n h), \\
& i=1,2,3, \quad B_{k_{i 21} l_{i 21}} u_{k_{i 21} l_{i 21}}=-0.08+0.06 \cos (8 \pi n h), \quad i=1,2,3 .
\end{aligned}
$$

The derived results of this paper are verified by the following steps.

(1) According to the illustrations of the neighbourhood distance $r$ for cell $C(k, l)=$ $C_{k_{i j h} l_{i j h}}$ which is given by $N_{\mathrm{r}}(i j h)$ function, and by (3.9) and (3.10), the exact values of distance $r$ and $\Omega$ are illustrated as:

$$
\begin{aligned}
& \Omega \triangleq\left\{x \in \mathcal{X} \subset N_{r}(i j h),\|x\|<\sum_{i=1}^{3} \sum_{j=1}^{2} \Theta_{i j}\right\}, \\
& \max _{n \in I_{N}}\left|x_{i j}[n]\right| \leq \max _{n \in I_{N}}\left\{\left|\varphi_{i j}[0]\right|+(1-\alpha(h))^{n} \sum_{h=1}^{1} \sum_{j=1}^{2}\left(\left|\bar{A}_{k_{i j h} l_{i j h}} y_{k_{i j h} l_{i j h}}[0]\right|\right)\right. \\
&\left.+(1-\alpha(h)) \sum_{h=1}^{1} \sum_{j=1}^{2}\left|\bar{B}_{k_{i j h} l_{i j h}} \bar{u}_{k_{i j h} l_{i j h}}\right|+\bar{I}_{i j}\right\} \triangleq \Theta_{i j}, \quad i=1,2,3 .
\end{aligned}
$$

Then, $\Theta_{i j}(i=1,2,3, j=1,2)$ is calculated below,

$$
\begin{aligned}
\max _{n \in I_{N}}\left|x_{1 j}[n]\right| \leq \max _{n \in I_{N}}\left\{\left|\varphi_{1 j}[0]\right|+(1-\alpha(h))^{n} \sum_{h=1}^{1} \sum_{j=1}^{2}\left(\left|\bar{A}_{k_{1 j h} l_{1 j h}} y_{k_{1 j h} l_{1 j h}}[0]\right|\right)\right. \\
\left.+(1-\alpha(h)) \sum_{h=1}^{1} \sum_{j=1}^{2}\left|\bar{B}_{k_{1 j h} l_{1 j h}} \bar{u}_{k_{1 j h} l_{1 j h}}\right|+\bar{I}_{1 j}\right\} \\
\leq 5.386+4.836+0.05 \times 4+0.24=10.662 \triangleq \Theta_{1 j}, \quad j=1,2,
\end{aligned}
$$




$$
\begin{gathered}
\max _{n \in I_{N}}\left|x_{2 j}[n]\right| \leq \max _{n \in I_{N}}\left\{\left|\varphi_{2 j}[0]\right|+(1-\alpha(h))^{n} \sum_{h=1}^{1} \sum_{j=1}^{2}\left(\left|\bar{A}_{k_{2 j h} l_{2 j h}} y_{k_{2 j h} l_{2 j h}}[0]\right|\right)\right. \\
+(1-\alpha(h)) \sum_{h=1}^{1} \sum_{j=1}^{2} \mid \bar{B}_{\left.k_{2 j h} l_{2 j h} \bar{u}_{k_{2 j h} l_{2 j h}} \mid+\bar{I}_{2 j}\right\}} \\
\leq 8.863+3.683+0.05 \times 4+0.24=12.986 \triangleq \Theta_{2 j}, \quad j=1,2, \\
\max _{n \in I_{N}}\left|x_{3 j}[n]\right| \leq \max _{n \in I_{N}}\left\{\left|\varphi_{3 j}[0]\right|+(1-\alpha(h))^{n} \sum_{h=1}^{1} \sum_{j=1}^{2}\left(\left|\bar{A}_{k_{3 j h} l_{3 j h}} y_{k_{3 j h} l_{3 j h}}[0]\right|\right)\right. \\
\left.+(1-\alpha(h)) \sum_{h=1}^{1} \sum_{j=1}^{2} \mid \bar{B}_{k_{3 j h} l_{3 j h}} \bar{u}_{\left.k_{3 j h} l_{3 j h} \mid+\bar{I}_{3 j}\right\}}\right\} \\
\leq 6.386+4.836+0.05 \times 4+0.24=11.662 \triangleq \Theta_{3 j}, \quad j=1,2 .
\end{gathered}
$$

Thus, the subset $\Omega$ of function $N_{r}(i j h)$ is derived by the following:

$$
\Omega \triangleq\left\{x \in X \subset N_{35.31}(i j h),\|x\|<\sum_{i=1}^{3} \sum_{j=1}^{2} \Theta_{i j}=35.31\right\} .
$$

(2) We will verify the condition of Theorem 3.1 if we want to utilize Theorem 4.1. After strictly calculating the condition of Theorem 3.1, it is easy to obtain that the function $g[n]=$ $\left|\widetilde{B}_{k_{i j 1} l_{i j 1}} u_{k_{i j 1} l_{i j 1}}\right|^{2}-\left|\widetilde{A}_{k_{i j} l_{i j}} y_{k_{i j 1} l_{i j}}\right|^{2}+\widetilde{I}_{i j}^{2} \in R^{+}, i=1,2,3, j=1,2, n \in I_{N}$; therefore, the condition of the Theorem 3.1 is critically satisfied as well.

(3) According to (4.1), the condition of the Theorem 4.1 will be derived as follows:

$$
\sum_{h=1}^{m_{h}} \sum_{j=1}^{m_{j}} \bar{A}_{k_{i j h} l_{i j h}}<\frac{3}{10}<\frac{1}{1 \times 1 \times 2}=\frac{1}{C_{y_{k l}} m_{h} m_{j}}
$$

Then state trajectories of neurons $x_{11}, x_{12}, x_{21}, x_{22}, x_{31}, x_{32}$ are shown in Figures 4 and 5.

From Figures 4 and 5, we can learn that all the periodic solution converges to a unique a $1 / 4 h$-periodic solution, then the DT-CNN (2.1) has a globally stable $1 / 4 h$-periodic solution. Thus, all conditions of Theorems 3.1 and 4.1 are strictly satisfied; therefore all conditions of proposed theorems are critically verified.

\section{Conclusions}

Existence and global stability are important dynamical properties in CNN. In this paper, we consider the discrete-time analogues of $\mathrm{CNN}$ with periodic coefficients and obtain some new 
State trajectories of the neurons $x_{11}$ and $x_{12}, h=1 / 4$.

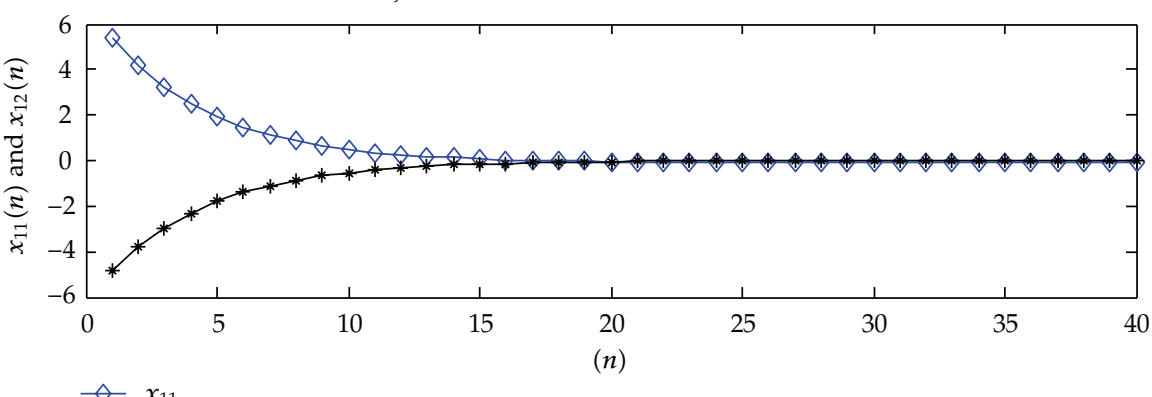

$\rightarrow-x_{12}$

State trajectories of the neurons $x_{21}$ and $x_{22}, h=1 / 4$.

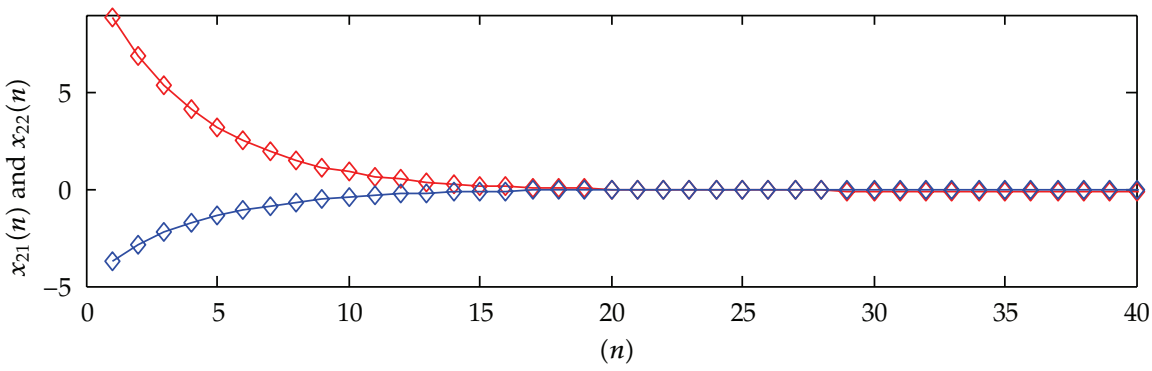

$\diamond x_{21}$

$\diamond x_{22}$

State trajectories of the neurons $x_{31}$ and $x_{32}, h=1 / 4$.

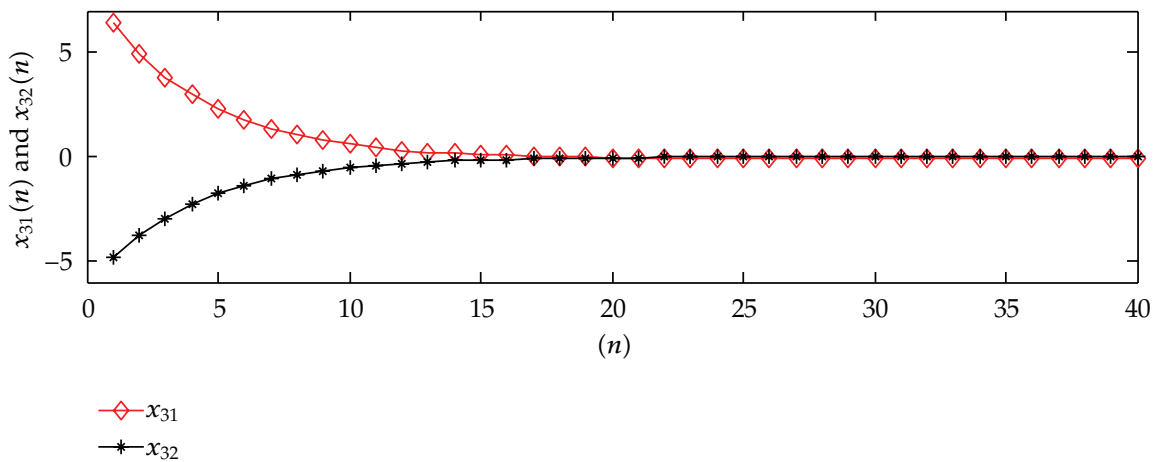

Figure 4: State trajectories of neurons $x_{11}, x_{12}, x_{21}, x_{22}, x_{31}, x_{32}(h=1 / 4)$.

results for the DT-CNN in the three-dimensional space. Comparisons between our results and the previous results have also been made. And it has been demonstrated that our criteria are more general and effective than those reported in the literature.

\section{Acknowledgments}

The authors wish to acknowledge Major Program of National Natural Science Foundation (NNSF) of China under Grants 11190015 and 60875035, Research Fund for the Doctoral 


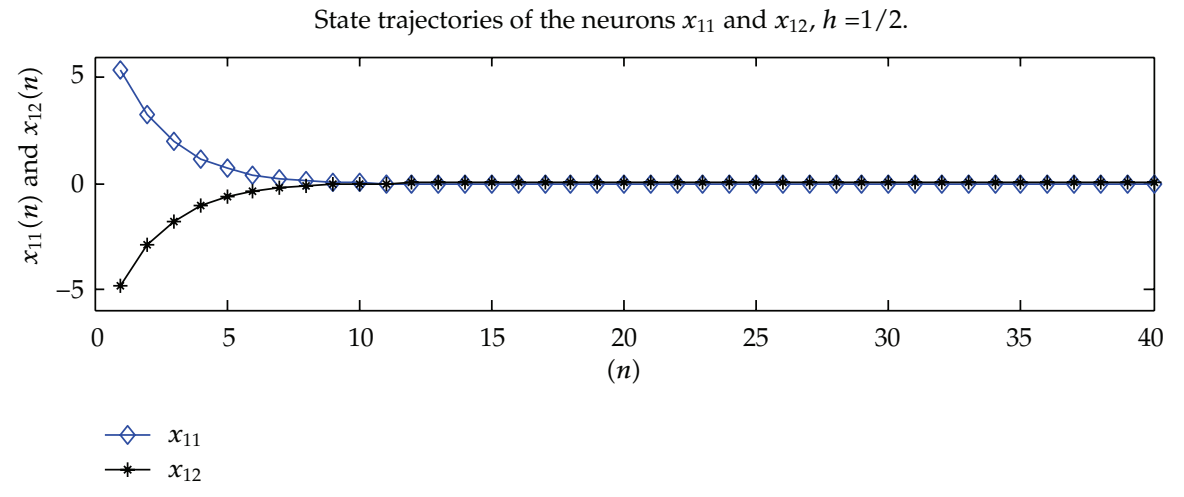

State trajectories of the neurons $x_{21}$ and $x_{22}, h=1 / 2$.

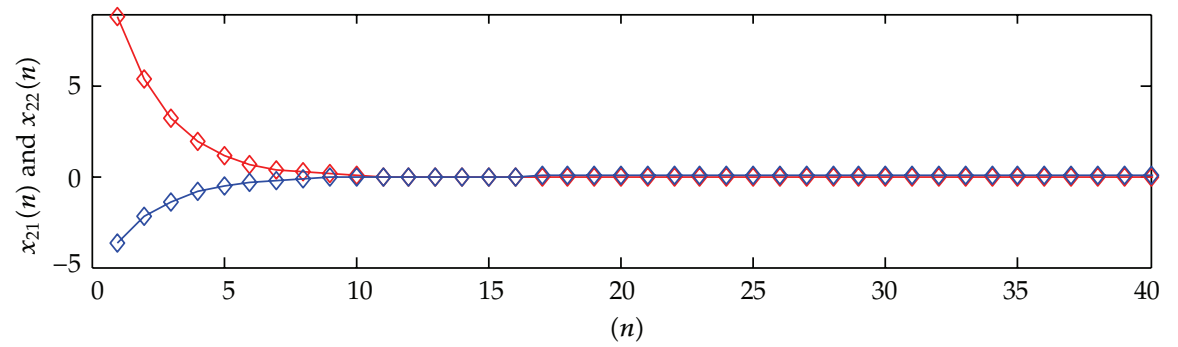

$\diamond x_{21}$

$\diamond x_{22}$

State trajectories of the neurons $x_{31}$ and $x_{32}, h=1 / 2$.

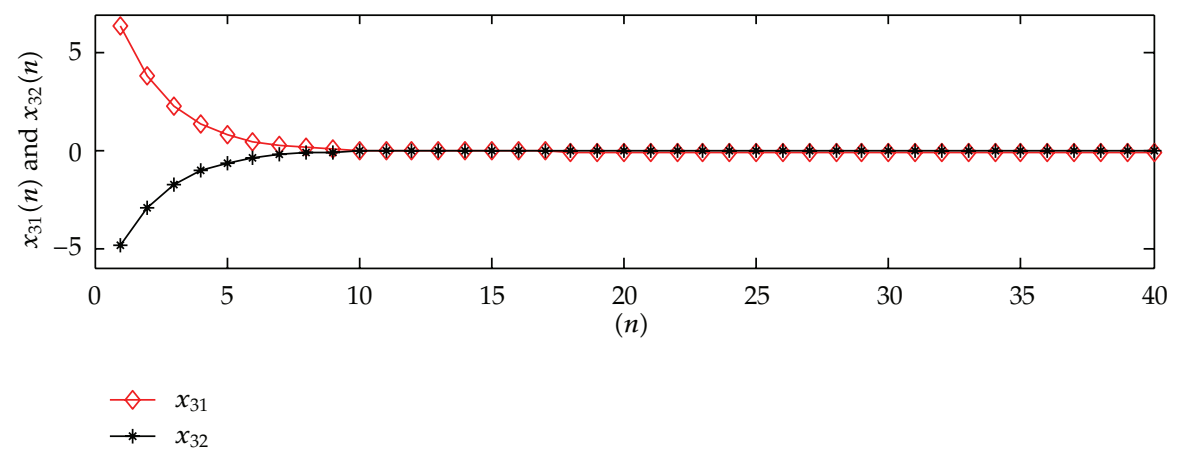

Figure 5: State trajectories of neurons $x_{11}, x_{12}, x_{21}, x_{22}, x_{31}, x_{32}(h=1 / 2)$.

Program of Higher Education of China under Grant 20100092110020, and Scientific Research Foundation of Graduate School of Southeast University under Grant YBJJ1215.

\section{References}

[1] L. O. Chua and L. Yang, "Cellular neural networks: theory," IEEE Transactions on Circuits and Systems, vol. 35, no. 10, pp. 1257-1272, 1988.

[2] Q. Zhang, L. Yang, and D. Liao, "Existence and exponential stability of a periodic solution for fuzzy cellular neural networks with time-varying delays," International Journal of Applied Mathematics and Computer Science, vol. 21, no. 4, pp. 649-658, 2011. 
[3] M. U. Akhmet, D. Aruğaslan, and E. Y1lmaz, "Stability in cellular neural networks with a piecewise constant argument," Journal of Computational and Applied Mathematics, vol. 233, no. 9, pp. 2365-2373, 2010.

[4] I. Stamova, H. Akca, and G. Stamov, "Qualitative analysis of dynamic activity patterns in neural networks," Journal of Applied Mathematics, vol. 2011, Article ID 208517, 2 pages, 2011.

[5] J. Javier Martinez, F. Javier Toledo, and J. Manuel Ferrandez, "Discrete-time cellular neural networks in FPGA," in Proceedings of the International Symposium on Field-Programmable Custom Computing Machines, pp. 293-294, 2007.

[6] P. Balasubramaniam, J. A. Samath, N. Kumaresan, and A. V. A. Kumar, "Solution of matrix Riccati differential equation for the linear quadratic singular system using neural networks," Applied Mathematics and Computation, vol. 182, no. 2, pp. 1832-1839, 2006.

[7] P. Balasubramaniam, J. Abdul Samath, and N. Kumaresan, "Optimal control for nonlinear singular systems with quadratic performance using neural networks," Applied Mathematics and Computation, vol. 187, no. 2, pp. 1535-1543, 2007.

[8] K. Mali and S. Mitra, "Symbolic classification, clustering and fuzzy radial basis function network," Fuzzy Sets and Systems, vol. 152, no. 3, pp. 553-564, 2005.

[9] P. Barmpalexis, F. I. Kanaze, K. Kachrimanis, and E. Georgarakis, "Artificial neural networks in the optimization of a nimodipine controlled release tablet formulation," European Journal of Pharmaceutics and Biopharmaceutics, vol. 74, pp. 316-323, 2010.

[10] J. Zhang, "Global stability analysis in delayed cellular neural networks," Computers E Mathematics with Applications, vol. 45, no. 10-11, pp. 1707-1720, 2003.

[11] W. Zhang and L. Wang, "Robust stochastic stability analysis for uncertain neutral-type delayed neural networks driven by Wiener process," Journal of Applied Mathematics, vol. 2012, Article ID 829594, 12 pages, 2012.

[12] J. Javier Martinez, F. Javier Toledo, and J. Manuel Ferrandez, “Implementation of a discrete cellular neuron model (DT-CNN) architecture on FPGA," Bioengineered and Bioinspired Systems II, vol. 5839, pp. 332-340, 2005.

[13] Y. Zhang, "Asymptotic stability of impulsive reaction-diffusion cellular neural networks with timevarying delays," Journal of Applied Mathematics, vol. 2012, Article ID 501891, 17 pages, 2012.

[14] Y. Li, "Global stability and existence of periodic solutions of discrete delayed cellular neural networks," Physics Letters A, vol. 333, no. 1-2, pp. 51-61, 2004.

[15] D. R. E. Gaines and J. L. Mawhin, Coincidence Degree and Non-Linear Differential Equations, Springer, Berlin, Germany, 1977. 


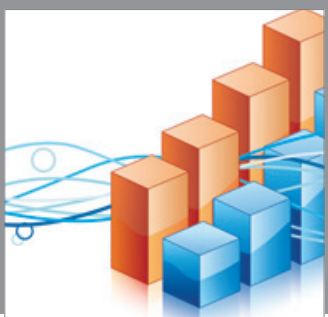

Advances in

Operations Research

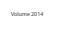

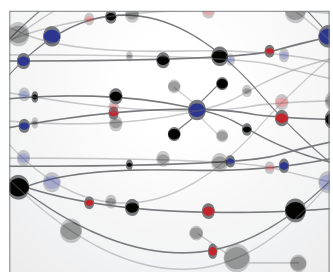

\section{The Scientific} World Journal
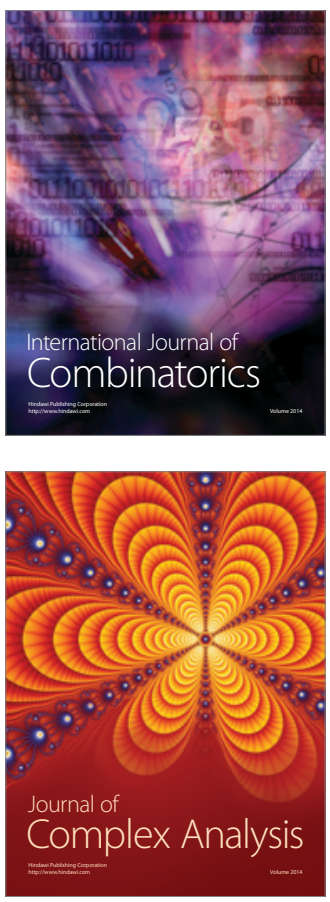

International Journal of

Mathematics and

Mathematical

Sciences
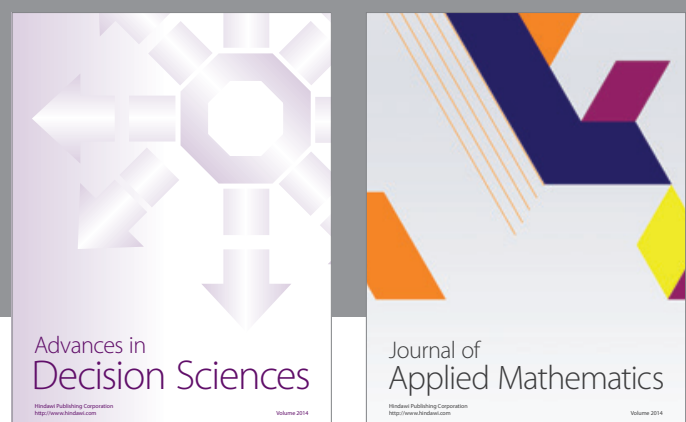

Journal of

Applied Mathematics
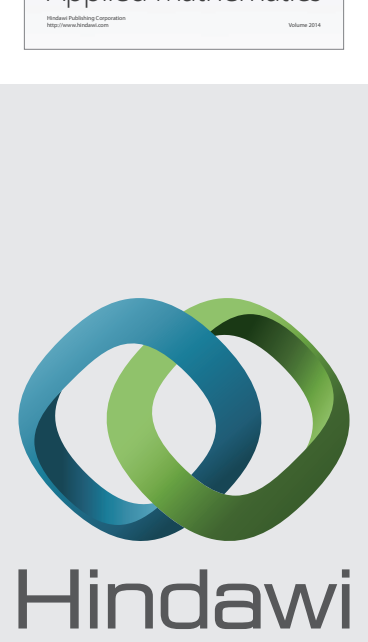

Submit your manuscripts at http://www.hindawi.com
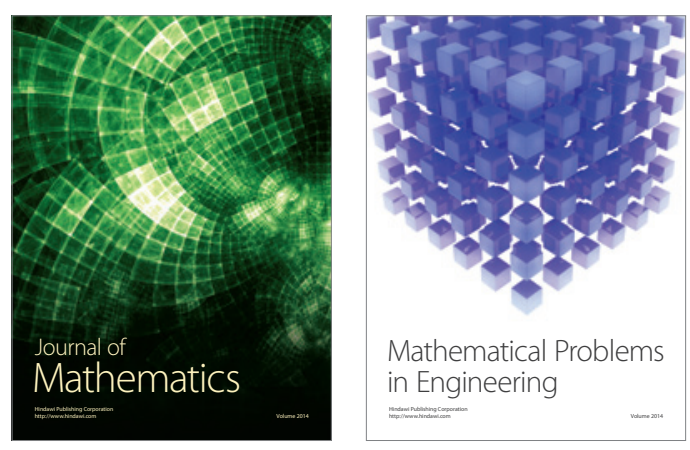

Mathematical Problems in Engineering
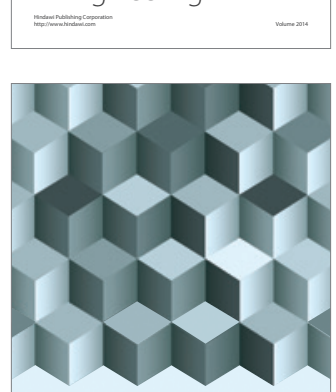

Journal of

Function Spaces
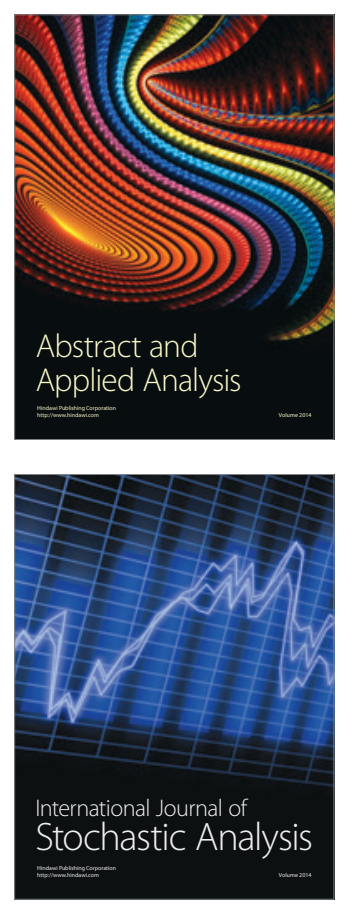

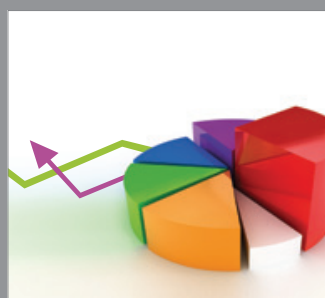

ournal of

Probability and Statistics

Promensencen
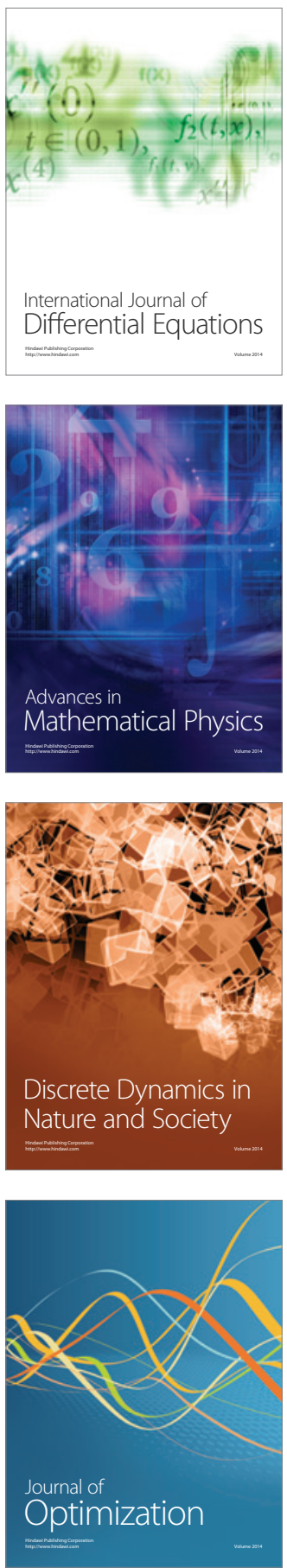